\title{
Anticipating the effects of visual gravity during simulated self-motion: estimates of time-to-passage along vertical and horizontal paths
}

\author{
Iole Indovina • Vincenzo Maffei · Francesco Lacquaniti
}

Received: 11 April 2013 / Accepted: 10 June 2013 / Published online: 27 June 2013

(C) Springer-Verlag Berlin Heidelberg 2013

\begin{abstract}
By simulating self-motion on a virtual rollercoaster, we investigated whether acceleration cued by the optic flow affected the estimate of time-to-passage (TTP) to a target. In particular, we studied the role of a visual acceleration $\left(1 \mathrm{~g}=9.8 \mathrm{~m} / \mathrm{s}^{2}\right)$ simulating the effects of gravity in the scene, by manipulating motion law (accelerated or decelerated at $1 \mathrm{~g}$, constant speed) and motion orientation (vertical, horizontal). Thus, 1-g-accelerated motion in the downward direction or decelerated motion in the upward direction was congruent with the effects of visual gravity. We found that acceleration (positive or negative) is taken into account but is overestimated in module in the calculation of TTP, independently of orientation. In addition, participants signaled TTP earlier when the rollercoaster accelerated downward at $1 \mathrm{~g}$ (as during free fall), with respect to when the same acceleration occurred along the horizontal orientation. This time shift indicates an influence of the orientation relative to visual gravity on response timing that could be attributed to the anticipation of the effects of visual gravity on self-motion along the vertical, but not the horizontal orientation. Finally, precision in TTP estimates was higher during vertical fall than when traveling at constant speed along the vertical orientation, consistent
\end{abstract}

I. Indovina $(\bowtie) \cdot$ V. Maffei $\cdot$ F. Lacquaniti Laboratory of Neuromotor Physiology, IRCCS Santa Lucia Foundation, Via Ardeatina 306, 00179 Rome, Italy

e-mail: i.indovina@hsantalucia.it

I. Indovina $\cdot$ V. Maffei $\cdot$ F. Lacquaniti

Centre of Space BioMedicine, University of Rome Tor Vergata,

Via Montpellier 1, 00133 Rome, Italy

F. Lacquaniti

Department of Systems Medicine, Neuroscience Section, University of Rome Tor Vergata, Via Montpellier 1,

00133 Rome, Italy with a higher noise in TTP estimates when the motion violates gravity constraints.

Keywords TTP · Vertical · Horizontal · Gravity · Visual · Self-motion

\section{Introduction}

To navigate in space, we must be able to calculate the time to passage to surrounding objects (time-to-passage, TTP). It is known that observers are able to estimate various self-motion parameters as rotation and translational heading from optic flow (for reviews Frost 2010; Britten 2008). Accelerated motion is frequent in Earth gravity $\left(1 \mathrm{~g}=9.8 \mathrm{~m} / \mathrm{s}^{2}\right)$, but the issue of discriminability of acceleration from visual information is still controversial. Most studies indicate that human and non-human primates can visually detect accelerations, but the discrimination of acceleration is much poorer than that of speed (Orban 2008; Brouwer et al. 2002; Werkhoven et al. 1992; Snowden and Braddick 1991; Calderone and Kaiser 1989; De Bruyn and Orban 1988). In some self-motion studies, TTP estimation did not seem to take into account the deceleration signal, while the use of acceleration signal appeared to depend on stimuli parameters (Festl et al. 2012; Capelli et al. 2010; Kaiser and Hecht 1995).

On the other hand, studies on interception of moving objects and on estimates of time intervals showed that a priori knowledge about gravitational acceleration, combined with realistic cues about the orientation and size of the visual scene, can influence response timing (Moscatelli and Lacquaniti 2011; Zago et al. 2009; Miller et al. 2008; Bosco et al. 2008; Senot et al. 2005; McIntyre et al. 2001). This a priori knowledge is thought to be stored in a visual-vestibular 
network of areas including the posterior-insula and the temporo-parietal junction (Indovina et al. 2013, 2005, Maffei et al. 2010; Miller et al. 2008; Bosco et al. 2008).

Here, we consider the possibility that also during visual self-motion, a priori knowledge about the gravitational acceleration may influence TTP estimate. The advantage for the brain of this a priori knowledge is evident in the estimation of time to impact when falling from a height, a problem faced during sports such as skiing, or at early stages of development. The problem of falling from a height is of prominent importance for non-human arboreal primates.

Here, we manipulated expectations about kinematics of self-motion by varying the orientation of motion along vertical and horizontal axes defined by the visual scene. We visually simulated rollercoaster rides along the vertical and horizontal orientations with accelerated $(1 \mathrm{~g})$, decelerated $(-1 \mathrm{~g})$ and constant speed motion. A previous study (Indovina et al. 2013) showed that these visual stimuli elicit comparable self-motion sensations across vertical and horizontal paths. In the present study, participants had to press a button at the time at which they thought the rollercoaster car would pass through a reference point ("Visible" protocol). If participants tuned their behavior based strictly on visual information about the motion law as derived by the optic flow, one would expect the same responses for vertical and horizontal self-motion for matched motion laws. On the other hand, if the visual context of self-motion (vertical or horizontal motion orientation) has an influence on the timing strategy employed by the participant, one might expect that subjects adjusted their responses depending on the direction of self-motion, in accordance with the expected direction of a gravitational force.

To time the response, participants could either calculate the TTP in an anticipatory manner from estimated motion parameters, or press the button when they detected a given proximity to the target. However, the kinematic parameter range could modify the response strategy across trials or conditions in an uncontrolled manner, for example, by inducing the selection of the anticipatory strategy in faster conditions and of the reactive strategy in slower conditions. To eliminate the confound generated by the use of a mixed strategy in the evaluation of response time, we performed an additional experiment ("Occluded" protocol) during which no visual information was provided during the last part of the path (Bosco et al. 2012; DeLucia and Liddell 1998). This manipulation forced participants to use only predictive estimates.

Our main finding was that, consistent with the hypothesis of an internal model storing a priori knowledge of gravitational acceleration, participants responded earlier during accelerated downward motion than during accelerated horizontal motion.

\section{Methods}

Participants

Ten right-handed subjects (4 males and 6 females, 26-42 years old, 31.8 years average age) with normal or corrected-to-normal vision gave written informed consent to participate. Each subject participated in two protocols ("Visible" and "Occluded"). In addition, eight of them also participated in a "self-motion perception" protocol. General procedures were approved by the Institutional Review Board of Santa Lucia Foundation, in conformity with the Declaration of Helsinki on the use of human subjects in research.

\section{Apparatus and task}

Participants sat in the dark in front of a PC monitor presenting visual stimuli that simulated rollercoaster rides (Fig. 1) across a mountain landscape (www.nolimitscoast er.com, Mad Data, Joerg Henseler, Erkrath, Germany) on a first-person perspective (see Indovina et al. 2013). AVI videos were displayed by means of Presentation 14.1 (Neurobehavioral Systems Inc., Albany, Canada), at $1024 \times 768$ pixels, 75 frames per second, $34 \times 27 \mathrm{~cm}, 80 \mathrm{~cm}$ distance from the screen corresponding to $24^{\circ} \times 19^{\circ}$ visual angle. Landscape elements as the mountains, the sky, the grass and the river provided cues to determine the scene orientation, while the optic flow parameters provided cues to kinematics. Participants' head leaned against the headrest of the armchair in vertical position. Participants were instructed to keep their head still. During a preparation time (2-6 s), the rollercoaster car reached the trial-specific initial position, velocity and orientation (heading vertically upward, downward or horizontally forward). This time period was introduced to be sure that participants were aware of the future motion orientation at trial onset. Afterward, the car traveled on rectilinear segments until a tunnel signaling the trial offset. At the entrance of the tunnel, there was a marked difference in the track color between outside and inside (see Fig. 1, track rails were white outside, black inside), well visible from the beginning of the trial. During the Visible protocol, participants were instructed to press a home-made button ( $1 \mathrm{~ms}$ resolution) when the car handlebar would cross the line with color change on the tracks at the entrance of the tunnel (see Fig. 1). Importantly, the visible scene during the last part of the motion was very similar for all conditions, the tunnel falling in the foveal visual field (see Fig. 1, right column). Participants were allowed to choose their gazing strategy. However, we assumed that the focus of expansion of the radial optic flow at the center of the screen represented the more natural fixed point of interest in common across conditions, due to its salience 


\section{Vertical Upward}

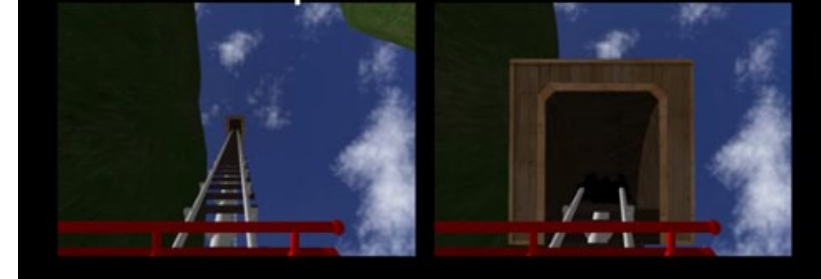

\section{Vertical Downward}

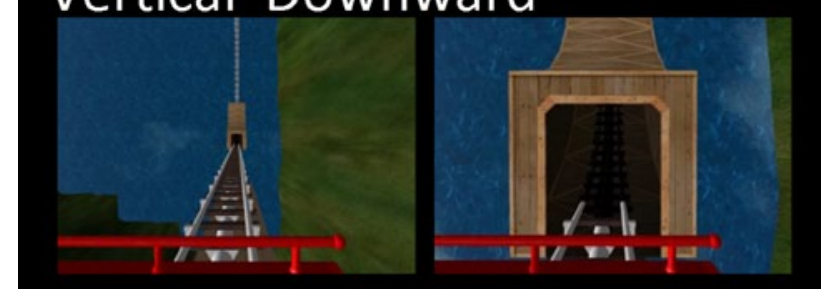

\section{Horizontal}

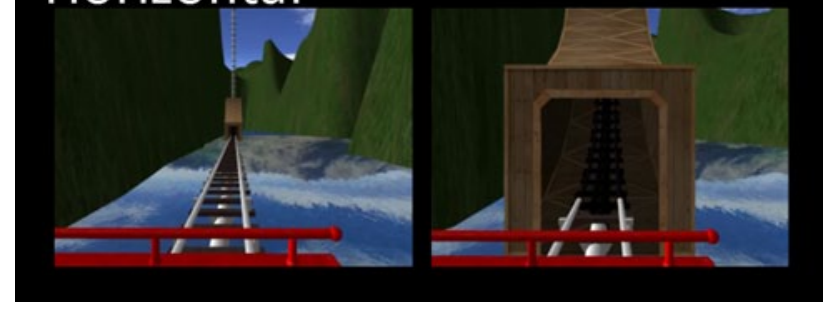

Fig. 1 Still frames from animated visual stimuli of the "Visible" protocol. Vertical and horizontal sections are shown at onset of the trial (left) and at about $2 \mathrm{~m}$ before crossing the passage reference point (right)

to elicit the behavioral response. This expansion focus coincided, at the beginning of each trial, with the end of the track at the tunnel entrance (see Fig. 1, left column). After target reaching, the visual motion was still present for $1 \mathrm{~s}$. During the Occluded protocol, instead, the visual scene disappeared for one of 3 different visual occlusion periods $(0.7,1,1.3 \mathrm{~s})$ before target reaching, being replaced by a black screen, and reappeared $0.2 \mathrm{~s}$ after target reaching. Participants had to press the button when they imagined the car would cross the reference point on the tracks. No performance feedback was provided (that is, whether the timing of the motor response was correct). This was done in order to investigate the contribution of internal feed-forward mechanisms in the absence of sensory error signals which may correct the performance with practice.

\section{Design}

The car moved at constant speed, constant acceleration $\left(9.8 \mathrm{~m} \mathrm{~s}^{-2}\right)$ or constant deceleration $\left(-9.8 \mathrm{~m} \mathrm{~s}^{-2}\right)$, depending on the trial. Acceleration or deceleration was consistent with visual gravity (and negligible friction) for vertical motion, so that downward motion was accelerated (as in
Table 1 Motion parameters characterizing each different trial for both horizontal and vertical orientations

\begin{tabular}{lrlll}
\hline $\begin{array}{l}\text { Trial } \\
\text { duration (s) }\end{array}$ & $L(\mathrm{~m})$ & $a$ & $d$ & $c$ \\
& & $\begin{array}{l}\text { V in/fin } \\
(\mathrm{Km} / \mathrm{h})\end{array}$ & $\begin{array}{l}V \text { in/fin } \\
(\mathrm{Km} / \mathrm{h})\end{array}$ & $V(\mathrm{Km} / \mathrm{h})$ \\
\hline 2.92 & 50 & $10 / 113$ & $113 / 10$ & 61 \\
4.01 & 90 & $10 / 152$ & $152 / 10$ & 81 \\
4.87 & 130 & $10 / 182$ & $182 / 10$ & 96 \\
\hline
\end{tabular}

$a$ accelerated, $d$ decelerated and $c$ constant speed. $L$ indicates the length of the rectilinear path of each trial. $V$ in/fin initial/final velocity at the offset of the trial

free fall) while upward motion was decelerated. Instead, the same acceleration or deceleration was consistent with powered propulsion for horizontal motion. The vertical condition simulated purely vertical displacements along the terrestrial vertical of the visual scene, in the absence of friction and horizontal perturbation forces that could cause the car to lose adherence with the tracks.

Overall, we crossed motion orientation (vertical, horizontal) and motion law ( $a=$ accelerated, $c=$ constant speed, $d=$ decelerated) resulting in seven conditions: vertical accelerated downward ( $\mathrm{Va} \downarrow$ ), vertical constant speed upward and downward $(\mathrm{Vc} \uparrow, \mathrm{Vc} \downarrow)$, vertical decelerated upward $(\mathrm{Vd} \uparrow)$, horizontal accelerated $(\mathrm{Ha})$, constant speed $(\mathrm{Hc})$ and decelerated $(\mathrm{Hd})$.

In order to avoid recognition of fixed time intervals, for each condition, we presented paths of three different durations (see Table 1 for the details about the kinematics). Constant speed during $\mathrm{Vc} / \mathrm{Hc}$ trials was the average velocity of the accelerated/decelerated trial of corresponding duration.

We presented each condition in blocks of 9 repetitions, randomizing durations and/or occlusion times within blocks. Blocks of the different conditions (7) were grouped in sessions separated by a few minutes rest. A total of 4 sessions randomizing condition blocks order were presented for each protocol, for a total of 36 trials per condition.

For each participant, the Occluded protocol was run about two weeks after the Visible protocol.

\section{Self-motion perception assessment}

Eight out of ten subjects participated to an additional experiment during which they were asked to rate the intensity of the self-motion perception. In particular, they had to rate "how strong was their sensation of being on the moving rollercoaster car" on a scale from 0 ("none") to 7 ("as on a real rollercoaster") during each of the seven conditions included in the Visible experiment. During the experiment, they kept the fingers (except the thumb) of each hand placed over 8 different buttons of a keyboard, so that they were ready to press 
the chosen key upon request. The movies were modified so that a green cross during each rectilinear section prompted the observer to rate his/her sensation of apparent self-motion by answering the question. To avoid effects related to handedness, the 0-to-7 order of the buttons was counterbalanced across participants (left to right or right to left). We correlated the self-motion rating results with TTP estimates across conditions in order to evaluate whether TTP estimation might be influenced by the intensity of self-motion perception.

\section{Behavioral data analysis}

TTP was calculated as the difference between the time participants pressed the button and the time the reference point was crossed. Outliers were excluded when they fell outside 1.5 times the interquartile range of a given participant and condition (typically $3 \pm 1 \%$ of all trials). Multivariate analysis of variance (MANOVA) was performed on TTP with motion orientation $(\mathrm{V} / \mathrm{H})$ and motion law $(\mathrm{a} / \mathrm{c} / \mathrm{d})$ as dependent variables, separately on each protocol. In addition, in the Occluded protocol, the occlusion time was added as a factor.

Finally, we studied the dependence on repetition across sessions. A significance level of 0.05 was used for all statistical tests. Post hoc paired $t$ tests were performed to investigate the nature of the significant effects of the MANOVA (Bonferroni corrected for multiple comparisons, denoted as $p$-corr). Data preprocessing was performed with Matlab 7.5.0 (The MathWorks Inc., Natick, MA) while MANOVAs and post hoc analyses were performed with Statistica (Statsoft).

If participants used only first-order information (velocity but not acceleration) to estimate TTP, we would expect that they estimated TTP better for constant speed motions, overestimated it for accelerated motions and underestimated it for decelerated motions. As a consequence, differences in TTP with respect to constant speed motions would be positive for accelerated and negative for decelerated motions. On the other hand, the use of both first- and second-order information (velocity and acceleration) would lead to an accurate response timing during all motions, corresponding to TTP non-different from zero. Finally, if participants used a priori information about gravity direction, we would expect that they differentiated TTP estimates during vertical motions with respect to the kinematically matched horizontal motions.

\section{Results}

Visible protocol

The difference in TTP between $\mathrm{Vc} \uparrow$ and $\mathrm{Vc} \downarrow$ conditions was not significant (paired $t$ test, $p=0.49$ ). Therefore, we pooled $\mathrm{Vc} \uparrow$ and $\mathrm{Vc} \downarrow$ in one condition denoted $\mathrm{Vc}$. A 2 (orientation) $\times 3$ (motion law) MANOVA showed a significant effect of motion law (Wilks' lambda test, $F(2,8)=9.4$, $p=0.007)$ indicating that the estimate of TTP differed according to the motion law. Indeed, TTP was underestimated for accelerated motions and overestimated for decelerated motions with respect to constant speed motions (paired $t$ test, $p$-corr $<0.03$ for all comparisons). Second, there was a significant orientation by motion law interaction (Wilks, $F(2,8)=7.6, p=0.013$ ).

Because there was no performance feedback, we focused on the relative differences in TTP (DTTP) between conditions. We found that DTTP was significantly negative for vertical accelerated motion versus horizontal accelerated motion (Fig. 2, Va vs. Ha, paired $t$ test, $p$-corr $=0.006$ ). Also the interaction of orientation by motion law restricted to accelerated and constant speed conditions was significant [(Va-Ha) vs. $(\mathrm{Vc}-\mathrm{Hc})$, paired $t$ test, $p$-corr $=0.018]$, while all remaining comparisons were not significant (all $p>0.3$, Fig. 2; Table 2). No significant effect of session was found for any of the conditions (all $F(3,8)<2.2$, all $p>0.1)$ in accordance with the expectation for a protocol without feedback.

These results indicate that, on average, participants anticipate the response during accelerated with respect to constant speed motions, and delay it during decelerated motion, independently of the motion orientation. The TTP dependence on orientation was evident during accelerated motions only, when the response was anticipated in the vertical with respect to the horizontal orientation.

For completeness, we also studied the effects of trial duration. The accelerated condition showed that TTP varied as a function of duration (duration short vs. long, $p$-corr $=0.009)$ while for constant and decelerated conditions duration short and long were not significantly different (all $p>0.1$ ). The difference between vertical and

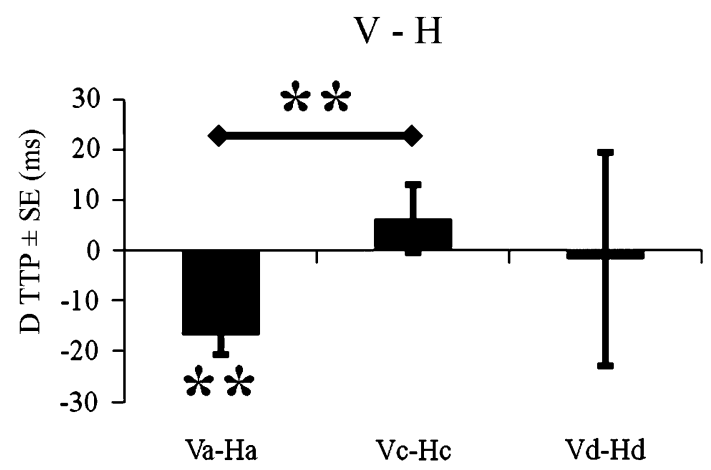

Fig. 2 Difference between TTP (DTTP, mean \pm SE) during vertical and horizontal motions as a function of motion law. Double asterisks indicate Bonferroni-corrected significant paired $t$ tests $(p$-corr $<0.05$, see Table 2) 
Table 2 Simple effects and direction orientation by motion law interactions during the Visible protocol

\begin{tabular}{lcll}
\hline & Mean \pm SE $(\mathrm{ms})$ & $p$ & $p$-corr \\
\hline Va-Ha & $-17 \pm 4$ & $\mathbf{0 . 0 0 2}$ & $\mathbf{0 . 0 0 7}$ \\
Vc-Hc & $6 \pm 7$ & 0.383 & n.s. \\
Vd-Hd & $-2 \pm 21$ & 0.938 & n.s. \\
(Va-Ha) versus (Vc-Hc) & $-23 \pm 6$ & $\mathbf{0 . 0 0 6}$ & $\mathbf{0 . 0 1 8}$ \\
(Vd-Hd) versus (Vc-Hc) & $-8 \pm 25$ & 0.758 & n.s. \\
(Va-Ha) versus (Vd-Hd) & $-15 \pm 22$ & 0.513 & n.s. \\
\hline
\end{tabular}

Mean values of TTP differences and corresponding $p$ values of statistical comparisons (two-tailed $t$ tests). Probability values are reported before and after Bonferroni correction ( $p$ and $p$-corr, respectively)

Significant values are highlighted in bold

SE standard error, n.s. non-significant

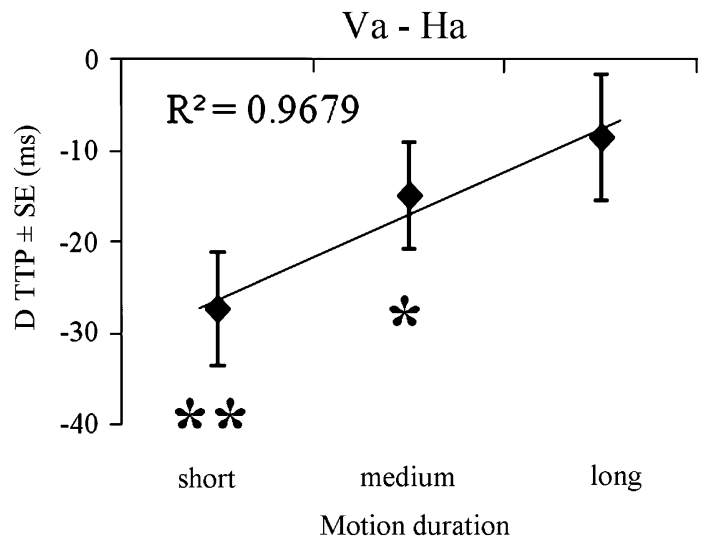

Fig. 3 Difference between TTP (DTTP) during accelerated vertical and horizontal motions as a function of motion duration (mean $\pm \mathrm{SE}$ ). Asterisks indicate values different from 0 at different levels of significance (two-tailed paired $t$ test, $* *$ Bonferroni-corrected $p$-corr $=0.015 ; * p=0.03$ uncorrected)

horizontal accelerated conditions decreased as the duration of the trial increased (linear regression slope $=9.4$, $p=0.036 F(1,19)=5.0)($ Fig. 3).

\section{TTP estimation precision}

We then examined the reliability of the TTP estimation mechanism as a function of the condition by analyzing the intra-individual variability. A 2 (orientation) $\times 3$ (motion law) MANOVA was performed on intra-individual variance. This analysis showed a significant main effect of the motion law $(F(2,8)=7.0, p=0.02)$. Post hoc comparisons showed increasing variance from accelerated to constant speed and from constant speed to decelerated conditions (all $p$-corr $<0.02$ Bonferroni corrected). This indicated that the precision during accelerated conditions was the highest; it was intermediate during constant speed and the lowest during decelerated conditions. However, this effect was

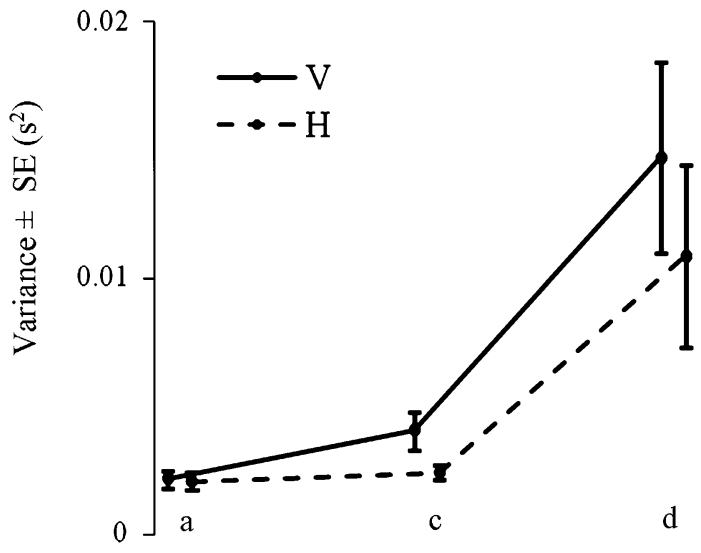

Fig. 4 Precision of the time-to-passage estimates: variance (mean $\pm \mathrm{SE}$ ) as a function of orientation (vertical/horizontal) and motion law ( $a$ accelerated, $c$ constant speed, $d$ decelerated)

mainly due to the vertical conditions. Indeed TTP precision during the vertical accelerated motion was higher than that during the vertical constant speed motion $(p$-corr $=0.05$ ), and showed a trend for the other comparisons (for precision during $\mathrm{Vc}$ greater than $\mathrm{Vd}, p=0.01$; for precision during Va greater than Vd, $p=0.007$ ) (see Fig. 4). Comparison between TTP precision during horizontal motions showed only a trend for $\mathrm{Ha}$ greater than $\mathrm{Hd}(p=0.04)$ and $\mathrm{Hc}$ greater than $\mathrm{Hd}(p=0.04)$. Finally, the constant speed condition showed a trend for greater precision in the horizontal with respect to the vertical TTP estimation $(p=0.04)$.

\section{Occluded protocol}

The Occluded protocol was performed in order to increase the contribution of non-visual information to the performance of the task. Results of the Occluded protocol were roughly in line with those of the Visible protocol. Because the comparison of TTP during $\mathrm{Vc} \uparrow$ versus $\mathrm{Vc} \downarrow$ was not significant ( $p=0.78$ ), we pooled the two conditions as Vc. A 2 (orientation) $\times 3$ (motion law) $\times 3$ (occlusion time) MANOVA showed a significant effect of orientation by motion law interaction $(F(2,8)=4.8, p=0.04)$. No significant effect of the occlusion time was found. Post hoc comparisons showed that only the interaction of orientation by motion law restricted to accelerated and constant speed conditions was significant ((Va-Ha) vs. (Vc-Hc), paired $t$ test, $p$-corr $=0.03$; Table 3 ). No significant effect of session was found for any of the conditions (all $F(3,8)<2.2$, all $p>0.1$ ).

\section{Self-motion perception assessment}

To control for the possible confound of differences in self-motion perception, we analyzed self-motion perception data acquired in a separate experiment on eight out of the ten subjects. MANOVA showed no significant effects 
Table 3 Simple effects and orientation by motion law interactions during the Occluded protocol

\begin{tabular}{lcll}
\hline & Mean \pm SE $(\mathrm{ms})$ & $P$ & $p$-corr \\
\hline Va-Ha & $-30 \pm 24$ & 0.24 & 0.72 \\
Vc-Hc & $26 \pm 19$ & 0.21 & 0.63 \\
Vd-Hd & $23 \pm 18$ & 0.24 & 0.72 \\
(Va-Ha) versus (Vc-Hc) & $-55 \pm 17$ & $\mathbf{0 . 0 1}$ & $\mathbf{0 . 0 3}$ \\
(Vd-Hd) versus (Vc-Hc) & $-27 \pm 23$ & 0.93 & n.s. \\
(Va-Ha) versus (Vd-Hd) & $52 \pm 28$ & 0.09 & 0.27 \\
\hline
\end{tabular}

Mean values of TTP differences and corresponding $p$ values of statistical comparisons (two-tailed $t$ tests). Probability values are reported before and after Bonferroni correction ( $p$ and $p$-corr, respectively)

Significant values are highlighted in bold

SE standard error, n.s. non-significant

(all $p>0.1$ ); on average, the self-motion rating was $4.2 \pm 0.3$ (mean $\pm \mathrm{SE}$ ). In particular, during the accelerated conditions, participants did not attribute a higher engaging value (sense of presence) to vertical with respect to horizontal motions (paired $t$ test, $p=0.14$ ). Correlation of sense of presence and TTP differences among vertical and horizontal accelerated conditions was not significant $(r=-0.05$, $p=0.9$; for both Visible and Occluded protocols).

Overall, the results of the control experiment showed that the sensation of apparent self-motion was adequate and comparable across all conditions.

\section{Discussion}

The purpose of this study was to examine whether there were differences in time-to-passage estimation due to the manipulation of self-motion orientation and motion law in relation to a visual gravity field. Participants virtually traveled on a rollercoaster and were asked to signal the arrival time to a target at the end of linear paths. Simulated self-motion occurred in the vertical or horizontal orientation with accelerated, decelerated or constant speed motion law. Only acceleration downward and deceleration upward was consistent with gravity-driven motion.

\section{Kinematics effect}

We found that, independently of the orientation of selfmotion, TTP showed a dependence on the motion law. In particular, participants anticipated on average the response during accelerated with respect to constant speed motions, and delayed it during decelerated motion. This behavior is in contrast with the predictions of the tau model (Tresilian 1999; 1995) extended to time-to-passage tasks (Festl et al. 2012; Capelli et al. 2010; Kaiser and Hecht 1995; Lee 1980, 1976), which assumes that second-order information (acceleration) is not used in predicting TTP. As a consequence, this classical model predicts that the duration of constant speed motions should be estimated correctly while it should be overestimated for accelerated and underestimated for decelerated motions. Previous studies were consistent in finding underestimation of decelerated motion indicating the use of first-order (velocity) information for this motion law (self-motion: Capelli et al. 2010; Kaiser and Hecht 1995; object motion: Maffei et al. 2010; Miller et al. 2008; Schlack et al. 2008; Indovina et al. 2005; Port et al. 1997). On the contrary, anticipated responses during accelerated motions and delayed responses during decelerated motions found in the current study suggest that participants used second-order information in the Visible protocol. The overestimation of positive and underestimation of negative accelerations correspond to an overestimate of the module of the acceleration vector. Also, the dependence of TTP on motion duration for the accelerated conditions is in contrast with the classical tau hypothesis. Indeed, this would predict that, given the same initial speed, the response to shorter duration trials should be more delayed than to longer duration trials, a trend opposite to our results.

\section{Vertical versus horizontal: accelerated self-motion}

We found a significant anticipation in the TTP estimate during the vertically accelerated downward motion (free fall) when compared with accelerated horizontal motion. As the motion law is the same for the two conditions, this difference must be related to the visual context that defines the orientation of the motion, vertical or horizontal. It can be concluded that the visual context has a priori influence on the motor response, possibly related to the expectation of an accelerated kinematics due to a gravitational force along the vertical orientation of the visual scene.

One alternative interpretation of this anticipation effect during vertical fall might be that it is a consequence of bigger arousal due to the potential risk associated with fall on a real rollercoaster. However, a previous study that used the same rollercoaster visual stimuli (Indovina et al. 2013) showed that participants did not attribute a bigger engaging value (sense of presence) to vertical with respect to horizontal accelerated motions. Here, in addition, we found no significant correlation between sense of presence and TTP difference among vertical and horizontal accelerated conditions.

It can be concluded that the difference found between accelerated vertical and horizontal TTP is compatible with the assumption that a different dynamics would underlie the motion along vertical and horizontal orientation. This conclusion is in line with previous observations on the interception of targets moving along different orientations (Senot et al. 2005). This previous study found that the interception timing for targets accelerating downwards 
was systematically anticipated relative to when the same targets accelerated upwards. This difference was abolished when the target travelled along the horizontal relative to the viewer (Senot et al. 2005), and was reversed during the weightless phases of parabolic flight, presumably due to altered influence of vestibular signals (Senot et al. 2012). We hypothesize that the high realism of the visual scene in our experiment caused the preponderance of visual over proprioceptive inputs (see Britten 2008). This was confirmed by the "sense of presence" experiment in which conditions with aligned or misaligned visual and proprioceptive inputs did not differ as for perceived realism.

Vertical versus horizontal: constant speed self-motion

In theory, a difference might be expected between the vertical and horizontal motion at constant speed as the gravitational a priori would predict an accelerated (decelerated) motion downward (upward) and so an anticipated (delayed) time response if the motion proceeds at constant speed, but is timed by the participant according to the gravity law. However, this difference was not detected in a previous study comparing upward and downward constant speed object motion (Senot et al. 2005) with participants in a fixed reference frame aligned with the Earth's vertical and the virtual vertical of the visual stimulation rotating accordingly with conditions as in the present experiment. Senot et al. (2005) found an anticipated (delayed) response during the downward (upward) motion only when varying the position of the head coherently with the condition (heading upward/downward), that is, adding the effect of gravity on vestibular and proprioceptive signal (De Saedeleer et al. 2013). In our study, we aimed to obtain an equivalent effect by adding realism to the scene. However, the lack of TTP difference found during constant speed conditions may be related to the better ability to judge constant velocities than constant accelerations as shown by the higher accuracy (success rate) found by Senot et al. (2005) during constant speed trials. Nonetheless, in the present study, we found that TTP estimation was more precise during horizontal than vertical condition at constant speed indicating that participants were more confident in timing horizontal constant speed motions. This is in line with the interpretation that constant speed motion is considered more likely along the horizontal than the vertical orientation. This result was even more evident when comparing vertical accelerated versus vertical constant speed motions. A previous study (Capelli et al. 2010) found an opposite trend for precision when participants estimated TTP during accelerated $\left(2.5 \mathrm{~m} / \mathrm{s}^{2}\right)$ and constant speed simulated self-motion along horizontal paths. This result is in line with the interpretation that $1-\mathrm{g}\left(9.8 \mathrm{~m} / \mathrm{s}^{2}\right)$ acceleration estimation may use a priori knowledge with respect to the arbitrary acceleration along the horizontal orientation used by Capelli et al. (2010).

Vertical versus horizontal: decelerated self-motion

If a priori information about gravity was used to estimate TTP during decelerated motion, we would have expected TTP to be overestimated during vertical with respect to horizontal decelerated motions. However, the lack of TTP difference in this comparison may have been related to the lower average velocity at which the target was reached with respect to the other conditions (see Table 1). The lower final speed may have rendered the use of a priori information unnecessary for TTP estimate during decelerated motions and induced the adoption of a reactive strategy to detect the target. Indeed, the higher variance related to this specific condition during the Visible protocol may suggest a conflict between the strategy adopted to perform the task (anticipatory vs. reactive).

In order to overcome this limit of the Visible protocol, we performed the Occluded protocol that prevented participants from using the reactive strategy. Nonetheless, also in this case, during the vertical decelerated condition, TTP was not significantly overestimated with respect to the horizontal condition. This was possibly due to the difficulty of the task that led to a high response variability.

In conclusion, we found that a priori knowledge of the gravity law plays a role by differentiating vertical and horizontal TTP responses to accelerated motions and its precision during vertical accelerated versus constant speed motions. Acceleration (positive or negative) appears to be taken into account but is overestimated in module in the calculation of TTP independently of orientation.

Acknowledgments Our work was supported by the Italian Ministry of Health (RF-10.057 grant), Italian Ministry of University and Research (PRIN grant) and Italian Space Agency (DCMC and CRUSOE grants).

Conflict of interest The authors declare no competing financial interests

\section{References}

Bosco G, Carrozzo M, Lacquaniti F (2008) Contributions of the human temporoparietal junction and MT/V5+ to the timing of interception revealed by transcranial magnetic stimulation. J Neurosci 28(46):12071-12084

Bosco G, Delle Monache S, Lacquaniti F (2012) Catching what we can't see: manual interception of occluded fly-ball trajectories. PLoS ONE 7(11):e49381

Britten KH (2008) Mechanisms of self-motion perception. Annu Rev Neurosci 31:389-410

Brouwer A-M, Brenner E, Smeets JBJ (2002) Perception of acceleration with short presentation times: can acceleration be used in interception? Percept Psychophys 64(7):1160-1168 
Calderone JB, Kaiser MK (1989) Visual acceleration detection: effect of sign and motion orientation. Percept Psychophys 45(5):391-394

Capelli A, Berthoz A, Vidal M (2010) Estimating the time-to-passage of visual self-motion: is the second order motion information processed? Vision Res 50(9):914-923

De Bruyn B, Orban GA (1988) Human velocity and direction discrimination measured with random dot patterns. Vision Res 28(12):1323-1335

De Saedeleer C, Vidal M, Lipshits M, Bengoetxea A, Cebolla AM, Berthoz A, Cheron G, McIntyre J (2013) Weightlessness alters up/down asymmetries in the perception of self-motion. Exp Brain Res 226(1):95-106

DeLucia PR, Liddell GW (1998) Cognitive motion extrapolation and cognitive clocking in prediction motion task. J Exp Psychol Hum Percept Perform 24(3):901-914

Festl F, Recktenwald F, Yuan C, Mallot HA (2012) Detection of linear ego-acceleration from optic flow. J Vis 12(7):1-12

Frost BJ (2010) A taxonomy of different forms of visual motion detection and their underlying neural mechanisms. Brain Behav Evol 75(3):218-235

Indovina I, Maffei V, Bosco G, Zago M, Macaluso E, Lacquaniti F (2005) Representation of visual gravitational motion in the human vestibular cortex. Science 308(5720):416-419

Indovina I, Maffei V, Pauwels K, Macaluso E, Orban GA, Lacquaniti F (2013) Simulated self-motion in a visual gravity field: sensitivity to vertical and horizontal heading in the human brain. Neuroimage 71:114-124

Kaiser MK, Hecht H (1995) Time-to-passage judgments in nonconstant optical flow fields. Percept Psychophys 57(6):817-825

Lee DN (1976) A theory of visual control of braking based on information about time-to-collision. Perception 5(4):437-459

Lee DN (1980) The optic flow field: the foundation of vision. Philos Trans R Soc Lond B Biol Sci 290(1038):169-179

Maffei V, Macaluso E, Indovina I, Orban G, Lacquaniti F (2010) Processing of targets in smooth or apparent motion along the vertical in the human brain: an fMRI study. J Neurophysiol 103(1):360-370
McIntyre J, Zago M, Berthoz A, Lacquaniti F (2001) Does the brain model Newton's laws? Nat Neurosci 4(7):693-694

Miller WL, Maffei V, Bosco G, Iosa M, Zago M, Macaluso E, Lacquaniti F (2008) Vestibular nuclei and cerebellum put visual gravitational motion in context. J Neurophysiol 99(4):1969-1982

Moscatelli A, Lacquaniti F (2011) The weight of time: gravitational force enhances discrimination of visual motion duration. J Vis 11(4):5, 1-17

Orban GA (2008) Higher order visual processing in macaque extrastriate cortex. Physiol Rev 88(1):59-89

Port NL, Lee D, Dassonville P, Georgopoulos AP (1997) Manual interception of moving targets. I. Performance and movement initiation. Exp Brain Res 116(3):406-420

Schlack A, Krekelberg B, Albright TD (2008) Speed perception during acceleration and deceleration. J Vis 8(8):9.1-9.11

Senot P, Zago M, Lacquaniti F, McIntyre J (2005) Anticipating the effects of gravity when intercepting moving objects: differentiating up and down based on nonvisual cues. J Neurophysiol 94(6):4471-4480

Senot P, Zago M, Le Séac'h A, Zaoui M, Berthoz A, Lacquaniti F, McIntyre $\mathrm{J}$ (2012) When up is down in $0 \mathrm{~g}$ : how gravity sensing affects the timing of interceptive actions. J Neurosci 32(6):1969-1973

Snowden RJ, Braddick OJ (1991) The temporal integration and resolution of velocity signals. Vis Res 31(5):907-914

Tresilian JR (1995) Perceptual and cognitive processes in time-tocontact estimation: analysis of prediction-motion and relative judgment tasks. Percept Psychophys 57(2):231-245

Tresilian JR (1999) Visually timed action: time-out for "tau"? Trends Cognit Sci (Regul. Ed.) 3(8):301-310

Werkhoven P, Snippe HP, Toet A (1992) Visual processing of optic acceleration. Vis Res 32(12):2313-2329

Zago M, McIntyre J, Senot P, Lacquaniti F (2009) Visuo-motor coordination and internal models for object interception. Exp Brain Res 192(4):571-604 